\title{
Insecticide treated nets use and its determinants among settlers of Southwest Ethiopia
}

Tsegaye Berkessa $^{1 *}$, D. Oljira ${ }^{1}$ and B. Tesfa ${ }^{2}$

\begin{abstract}
Background: Ethiopia is rapidly increasing insecticide-treated nets (ITNs) coverage to combat malaria, but adequate follow-up and factors affecting use of ITNs is lacking. The aim of this study was to assess determinants of the use of ITNs in a southwest area of Ethiopia.

Methods: This cross-sectional survey was conducted in the Chewaka district settlement area of southwest Oromia from March to May, 2013. Kebeles were stratified by degree of urbanization (rural, peri-urban, or urban). Randomly selected households, which had been freely supplied with at least one ITN, were surveyed using a pre-tested, structured questionnaire administered through household interviews. Logistic regression analysis was used to examine the association between use of ITNs and determinant factors.

Results: Of 574 households surveyed, $72.6 \%$ possessed ITNs and $80 \%$ of these had been used the night before the survey. The most common reasons for the absence ITNs in the household identified in this study were ITNs were old and therefore discarded and that households use ITNs for purposes other than their intended use. The multivariate analysis found that knowledge of malaria transmission by mosquito bites (Adjusted OR=3.44, $95 \% \mathrm{Cl}$ : 1.80-6.59), and washing of ITNs at least once by households (Adjusted OR=2.66, $95 \% \mathrm{Cl}$ : 1.35-5.26) were significantly associated with an ITN being used by households. The mean possession was 1.59 ITN per household (3.57 persons per an ITN). One hundred fifty four (36.9 \%) of ITNs had at least one hole/tear. Among these, 108 (70.1 \%) ITNs had at least one hole/tear with greater than $2 \mathrm{~cm}$ and 29 (18.8\%) had greater than seven holes/tears.

Conclusions: This study in Southwest Ethiopia showed a high proportion of net ownership compared to a household survey from Ethiopia which included in the World Malaria Report. Despite somewhat high percentages ITN ownership, the study demonstrated there was still a gap between ownership and use of ITNs. Use of ITNs was affected by knowledge of malaria transmission by mosquito bite and washing of ITNs at least once by households. Intensive health education and community mobilization efforts should be employed to attempt to influence these factors that significantly affect ITN use.
\end{abstract}

Keywords: ITN utilization, Settlers, Malaria prevention, Ethiopia

\footnotetext{
* Correspondence: tsegayebtola@gmail.com

'Department of Public Health, Faculty of Public Health and Medical Sciences,

Mettu University, Mettu, PO Box 318, llu Ababor, Ethiopia

Full list of author information is available at the end of the article
} 


\section{Background}

Malaria remains a major public health problem particularly in sub-Saharan Africa. In 2012, malaria was responsible for over 1.1 million deaths globally [1] and was endemic in 104 countries with substantial geographic disparities. Around $81 \%$ of the malaria incidence and $91 \%$ of the malaria deaths in 2010 occurred in Africa [2]. Approximately 52 million people in Ethiopia (approximately $68 \%$ of the national population) live in malaria risk areas, primarily at altitudes below $2000 \mathrm{~m}$ [3]. In Oromia $75 \%$ of the land is considered malarious, accounting for over 17 million people at risk of infection [4].

Insecticide-treated bed nets (ITNs) are a means of malaria control and prevention [5]. The impact of ITNs on reducing malaria episodes is well documented $[6,7]$. Use of ITNs is one of the major vector control measures in Ethiopia. More than 20 million ITNs were distributed between 2005 and 2007, enabling $68 \%$ of the households living in malaria-endemic areas to own at least one ITN. Indeed, 15 million ITNs were distributed in 2010 and 2011 to replace long-lasting insecticidal nets (LLINs) distributed previously $[3,8]$. Beyond household possession of ITNs, it is crucial to understand household use of ITNs. The ITNs that are available at a household level may be left unused; or alternatively, even if ITNs are used, usage may be intermittent and/or vulnerable members of the household may not be given priority. The maximum malaria reduction impact of ITNs will only be achieved if people acquire nets, treat/re-treat them, make sure that the most vulnerable household members sleep under them, and use nets through the year [9].

Studies in various African countries, including Ethiopia, have revealed discrepancies between ITN possession and use $[8,10-12]$. Yet, there is no properly documented evidence regarding the coverage and use of ITNs among settlers in malaria-prone areas. This study was designed to investigate the possession, use, and factors affecting use of ITNs in the Chewaka district settlement area. This study also helped to evaluate the local ITN programs with reference to the Abuja targets.

\section{Methods}

\section{Study setting and design}

This cross sectional survey was conducted in Chewaka district settlement, located approximately $566 \mathrm{~km}$ from Addis Ababa in the Southwest Oromia Region of Ethiopia. It has a total population of 65,850 , with people residing in 28 kebeles. The climatic condition of the area is a tropical zone referred to as 'cola'. It accounts $54.22 \mathrm{~km}^{2}$ of land area which is all the land masses are suitable for settlement. The annual temperature and rainfall varies from 37 to $40^{\circ} \mathrm{C}$ and 1000 to $1200 \mathrm{~mm}$ respectively, and the altitude ranges from 900 to $1400 \mathrm{~m}$ above sea level. There is known the successful settlement in Oromia and by sesame seed production.
The main rivers in the area include the Didesa and Dabana, which are tributaries of the Abay River. In the district there are three health centers, twenty seven health posts, eighteen clinics and one drug vendor (Chewaka district annual health office report, unpublished).

\section{Sample size and sampling technique}

The sample size for the survey was calculated by using the formula for a single population proportion, including a $95 \%$ CI, $5 \%$ margin of error, and estimate of $73 \%$ of household using ITNs based on a previous study in Ethiopia [13]. With a $5 \%$ adjustment for non-response rate and a design effect of 2 , the resulting calculation for a total sample size was 636 households. The study employed a multi-stage sampling technique, taking into consideration that socio-demographic factors affecting ITN use might differ based on the household's distance from the district town. The 28 malarious kebeles of the district were first stratified into three groups (urban, semi-urban, and rural kebeles), and then six kebeles were randomly selected by lottery method by proportional allocation to size ( 1 from the first, 2 from the second and 3 from the third stratum). Health extension workers (HEWs) distributed ITNs to the community at their vicinity/households during free massdistribution campaigns. Households were randomly selected from a list provided by the district administration

\section{Data collection method and analysis}

Data were collected by diploma health professionals after training using a pretested, structured questionnaire prepared in English and then translated into the local language of Afan Oromo. The questionnaire was adopted from instruments developed by the Roll Back Malaria (RBM) partnership Monitoring and Evaluation Reference Group by the WHO and UNICEF [14]. Data collection was conducted during the peak malaria transmission period, from March to May, 2013. Data collectors administered the questionnaire through household visits. Information was primarily collected from the head of households (father or mother), or if this was not possible, from another adult household member. The condition of household nets was inspected by use of a checklist. Data was entered using Epidata version 3.1 and Stata version 11.0 was used for analysis. Descriptive statistics provided means and percentages related to socio-demographic characteristics; knowledge of malaria transmission and prevention; and possession, use, and condition of ITNs. Through logistic regression, adjusted odds ratios were calculated to identify predictors of ITN use.

\section{Ethical consideration}

Ethical clearance was obtained from the Mettu University Faculty of Public Health and Medical Sciences Institutional 
Research Ethics Review Committee, as well as from the zonal and woreda-level health offices. Before each interview, researchers sought consent from each respondent.

\section{Results}

Socio demographic characteristics

A total of 574 households participated in this study with a response rate of $90.3 \%$, of which 377 (65.7\%) were heads of households. The majority of the respondents $352(61.3 \%)$ were female. The mean (SD) age of the respondents was $30.6(8.9)$ years and the mean (SD) family size was 5.7 (2.2). Of the total households, 470 (81.9\%) had at least one child under 5 years of age (in total 703) and $86(15 \%)$ had pregnant women (one in each household). A majority of respondents (379 or $66 \%$ ) were illiterate (unable to read and write) and 414 (72.1\%) were farmers (Table 1).

\section{Knowledge of respondents about malaria transmission and prevention}

From the total respondents, 342 (59.6 \%) mentioned mosquitoes bite as the main mode of transmission for malaria. The other means of transmission reported included (232, $40.4 \%)$ : living near stagnant water $(138,24.0 \%)$, feeling cold (16, $2.7 \%)$, presence of waste $(35,6.1 \%)$, drinking dirty water $(24,4.2 \%)$, being hungry and being in the rain (both $9,1.6 \%$ respectively). The majority of respondents $(208,39.3 \%)$ reported ITNs as the main preventive measure against malaria; followed by proper waste disposal $(89,16.8 \%)$, taking tablets $(85,16.1 \%)$, use of aerosol sprays (59,11.2 \%), drainage of breeding sites (59,11.2 \%), use of traditional remedies $(19,3.6 \%)$, and fumigation $(9,1.7 \%)$. The majority $(495,86.2 \%)$ of respondents had ever heard/seen messages about ITNs. Most (56598.4 \%) of the respondents believed that sleeping under an ITN is beneficial, and only 42 (7.3 \%) respondents reported problems associated with sleeping under an ITN (Table 2).

\section{ITN possession and use}

All households $(n=574)$ that participated in this study were freely provided with at least one ITN by the district health office. Two hundred ninety seven (51.7 \%) households were supplied with one, 215 (37.5\%) with two, 61 $(10.6 \%)$ with three ITNs and $1(0.2)$ with four ITNs. In total, 914 ITNs were supplied to the households included in this study. Of these, 731 (80\%) were reported as being used by households. Use varied among strata at $75 \%, 84.9 \%$ and $82.7 \%$ for rural, semi urban and urban strata respectively. Among ITNs that were used, about $37.4 \%$ had at least one hole. Mean possession was 1.59 ITNs per household (3.57 people per an ITN).

ITNs were not found in 157 (27.4\%) households at the time of the survey. The reasons for absence of ITNs
Table 1 Socio-demographic characteristic of respondents in Chewaka district, South West Ethiopia, 2014

\begin{tabular}{|c|c|c|}
\hline Variables $(n=574)$ & Frequency & Percent \\
\hline \multicolumn{3}{|l|}{ Sex } \\
\hline Male & 222 & 38.7 \\
\hline Female & 352 & 61.3 \\
\hline \multicolumn{3}{|l|}{ Age (years) } \\
\hline $15-29$ & 286 & 49.8 \\
\hline $30-44$ & 237 & 41.3 \\
\hline$\geq 45$ & 51 & 8.9 \\
\hline \multicolumn{3}{|l|}{ Marital Status } \\
\hline Single & 50 & 8.7 \\
\hline Married & 507 & 88.3 \\
\hline Divorced & 10 & 1.8 \\
\hline Widowed & 7 & 1.2 \\
\hline \multicolumn{3}{|l|}{ Educational level } \\
\hline Illiterate & 379 & 66 \\
\hline Literate & 195 & 34 \\
\hline \multicolumn{3}{|l|}{ Occupation } \\
\hline Student & 31 & 5.4 \\
\hline House wife & 70 & 12.2 \\
\hline Governmental Employee & 19 & 3.3 \\
\hline Merchant & 35 & 6.1 \\
\hline Farmer & 414 & 72.1 \\
\hline Other & 5 & 0.9 \\
\hline \multicolumn{3}{|l|}{ Monthly income of HH (birr) } \\
\hline$<100$ & 13 & 2.3 \\
\hline 100-299 & 129 & 22.5 \\
\hline $300-499$ & 167 & 29.1 \\
\hline $500-799$ & 149 & 26 \\
\hline$>799$ & 116 & 20.2 \\
\hline \multicolumn{3}{|l|}{ Family Size } \\
\hline Less than three & 35 & 6.1 \\
\hline Three to four & 158 & 27.5 \\
\hline Five to six & 179 & 31.2 \\
\hline Seven and above & 202 & 35.2 \\
\hline \multicolumn{3}{|l|}{ Presence of radio in the $\mathrm{HH}$} \\
\hline No & 293 & 51.1 \\
\hline Yes & 281 & 48.9 \\
\hline \multicolumn{3}{|c|}{ Presence of television in the $\mathrm{HH}$} \\
\hline No & 544 & 94.8 \\
\hline Yes & 30 & 5.2 \\
\hline
\end{tabular}

included: lost or stolen $(24,15.3 \%)$, used for other purposes (e.g. storage of sorghum, to make fences, protect bulls from insect bites) $(46,29.3 \%)$, and thrown away as old $(84,53.5 \%)$, and gave to others (3, $1.9 \%)$.In addition, 
Table 2 Knowledge of respondents about the transmission mechanism and preventive measures of malaria, ITN awareness and associated factors in Chewaka district, South West Ethiopia, 2014

\begin{tabular}{|c|c|c|}
\hline Variables & Frequency & Percent \\
\hline \multicolumn{3}{|c|}{ Main transmission mechanism of malaria $(n=574)$} \\
\hline Bitten by mosquito & 342 & 59.6 \\
\hline Living near collected water & 138 & 24.0 \\
\hline Feeling cold & 16 & 2.7 \\
\hline Presence of wastes & 35 & 6.1 \\
\hline Drinking dirty water & 24 & 4.2 \\
\hline Being hungry & 9 & 1.6 \\
\hline Being in the rain & 9 & 1.6 \\
\hline Other & 1 & 0.2 \\
\hline \multicolumn{3}{|l|}{ Main preventive measures of malaria $(n=529)$} \\
\hline Use of ITN & 208 & 39.3 \\
\hline Take tablet & 85 & 16.1 \\
\hline Proper disposal of wastes & 89 & 16.8 \\
\hline Use of traditional remedies & 19 & 3.6 \\
\hline Fumigants & 9 & 1.7 \\
\hline Use insecticide spray & 59 & 11.2 \\
\hline Drainage & 59 & 11.2 \\
\hline Others & 1 & 0.2 \\
\hline \multicolumn{3}{|c|}{ Ever heard education messages about ITNs $(n=574)$} \\
\hline No & 79 & 13.8 \\
\hline Yes & 495 & 86.2 \\
\hline \multicolumn{3}{|c|}{ Think that sleeping under ITN have benefit $(n=574)$} \\
\hline No & 9 & 1.6 \\
\hline Yes & 565 & 98.4 \\
\hline \multicolumn{3}{|l|}{ The benefits of sleeping under ITN $(n=565)$} \\
\hline Don't get bitten by mosquito & 274 & 48.5 \\
\hline Don't get bothered by other mosquito & 95 & 16.8 \\
\hline Don't get malaria & 191 & 33.8 \\
\hline To get warmth & 5 & 0.9 \\
\hline \multicolumn{3}{|c|}{ Believe that sleeping under ITN has problem $(n=574)$} \\
\hline No & 532 & 92.7 \\
\hline Yes & 42 & 7.3 \\
\hline \multicolumn{3}{|c|}{ Problems associated with sleeping under ITNs $(n=42)$} \\
\hline Difficult to get at night & 18 & 42.9 \\
\hline It is too hot to sleep under ITNs & 14 & 33.3 \\
\hline It takes time to tuck a net each night & 5 & 11.9 \\
\hline Mosquito can still bite through ITN & 4 & 9.5 \\
\hline No enough air when sleeping under & 1 & 2.4 \\
\hline
\end{tabular}

20 (4.8\%) of the households did not use their nets due to: hot to sleep under the net $(7,35 \%)$, housing construction problems (lack of appropriate place for hanging the net)
(6, $30 \%)$, absence of mosquitoes (2, $10 \%)$, fear of its toxicity (2, $10 \%)$, absence of bed (2, $10 \%)$, and perception that ITN could not prevent malaria (1, $5 \%)$. This implies that 334 (80 \%) of the households used at least one of their freely supplied ITNs. The night before the day of the survey, $77.4 \%$ (418) of all children under five and $75 \%$ (54) of all pregnant women slept under an ITN (Table 3).

\section{The condition of ITNs}

Among households who owned ITNs $(n=417)$ at the time of the survey, $305(73.1 \%)$ had been washed at least once and100 (24\%) had been washed three or more times. One hundred fifty four (36.9\%) ITNs had at least one hole/tear. Among these, 29 (18.8 \%) ITNs had greater than seven holes/tears and 108 (70.1 \%) ITNs had at least one hole/tear greater than $2 \mathrm{~cm}$ (Table 4).

\section{Determinants of ITN utilization}

Factors associated with use of at least one ITN by households were knowledge of malaria transmission by mosquito bite (Adjusted OR = 3.44, 95 \% CI: 1.80-6.59), and ITNs washed at least once by household (Adjusted OR = 2.66, 95 \% CI: 1.35-5.26). Sex and age of respondents, number of ITNs freely supplied, presence of children under five / any children in the household, and age of ITNs were not associated with the use of ITNs by households, when use was adjusted for the other factors (Table 5).

\section{Discussion}

The study showed that ownership and use of ITNs in the study area were $72.6 \%$ and $80.1 \%$ respectively. The percentage of children under 5 years of age and pregnant women not using ITNs exceeded that of other adults. The two factors strongly associated with use of ITNs the night before the survey were knowledge of malaria transmission by mosquito bites and ITNs being washed at least once by households.

The proportion of households possessing ITNs was higher in this study compared to the average figure indicated in World Malaria Report of 2011 (median = 56 \%) from household survey results [15]. This implies that the net distribution program is going well when compared to the Roll Back Malaria [16] and World Health Assembly targets [17]. In this study, use of ITNs was in line with WHO recommendation of $80 \%$ utilization [15]. High rates of use achieved within a short period of time demonstrates acceptance of nets by users as a major malaria control tool and reflects the concerted efforts of the Ministry of Health. However, more than half of households had just a single net, and on average four individuals shared a single net. This issue requires great attention, because the national policy aims to provide one ITN for every sleeping space (approximately one net per 
Table 3 ITN possession and utilization by households in Chewaka district, South West Ethiopia, 2014

\begin{tabular}{|c|c|c|c|c|c|}
\hline & \\
\hline Variables & Frequency & Percent & Variables & Frequency & Percent \\
\hline \multicolumn{3}{|l|}{ Number of ITNs freely supplied for HHs } & \multicolumn{3}{|l|}{ Age of ITNs $(n=417)$} \\
\hline One/HH & 297 & 51.7 & $<1$ year & 188 & 45.1 \\
\hline $\mathrm{Two} / \mathrm{HH}$ & 215 & 37.5 & $1-2$ years & 126 & 30.2 \\
\hline Three/HH & 61 & 10.6 & $>3$ years & 103 & 24.7 \\
\hline Four/HH & 1 & 0.2 & Shape $(n=417)$ & & \\
\hline \multicolumn{3}{|l|}{ Utilization of ITNs by HHs } & Rectangular & 413 & 99 \\
\hline Currently used & 334 & 80.1 & Conical & 4 & 1 \\
\hline Not used & 83 & 19.9 & Color $(n=417)$ & & \\
\hline \multicolumn{3}{|c|}{ Availability of at least one freely supplied ITNs } & White & 4 & 1 \\
\hline No & 157 & 27.4 & Green & 143 & 34.3 \\
\hline Yes & 417 & 72.6 & Blue & 270 & 64.7 \\
\hline \multicolumn{3}{|l|}{ Reason for unavailability } & \multicolumn{3}{|c|}{ ITNs ever been washed $(n=417)$} \\
\hline Lost/stolen & 24 & 15.3 & Yes & 305 & 73.1 \\
\hline Used for other purpose & 46 & 29.3 & No & 112 & 26.9 \\
\hline Old then thrown away & 84 & 53.5 & \multicolumn{3}{|c|}{ Frequency of washing $(n=305)$} \\
\hline Given to others & 3 & 1.9 & One to three times & 232 & 76.1 \\
\hline \multicolumn{3}{|c|}{ Households reported use at least one of their available ITNs } & Four to six times & 50 & 16.4 \\
\hline No & 20 & 4.8 & Seven or more times & 23 & 7.5 \\
\hline Yes & 397 & 95.2 & \multicolumn{3}{|c|}{ Presence of hole/tear on ITN $(n=417)$} \\
\hline \multicolumn{3}{|l|}{ Frequency of using their ITNs } & Yes & 154 & 36.9 \\
\hline Consistently throughout the year & 278 & 70 & No & 263 & 63.1 \\
\hline Intermittently & 119 & 30 & \multicolumn{3}{|c|}{ Number of holes/tears $(n=154)$} \\
\hline \multicolumn{3}{|l|}{ Time they use intermittently $(n=119)$} & $1-7$ & 125 & 81.2 \\
\hline During rain & 92 & 77.3 & $>7$ & 29 & 18.8 \\
\hline After rain & 7 & 5.9 & \multicolumn{3}{|c|}{ Size of holes/tears $(n=154)$} \\
\hline As they like & 2 & 1.7 & $\leq 2 \mathrm{~cm}$ & 46 & 29.9 \\
\hline When hearing mosquito buzzing & 18 & 15.1 & $>2 \mathrm{~cm}$ & 108 & 70.1 \\
\hline
\end{tabular}

Reason for not using the available ITNs $(n=20)$

$\begin{array}{lll}\text { Absence of mosquito } & 2 & 10 \\ \text { Absence of bed } & 2 & 10 \\ \text { ITNs do not prevent malaria } & 1 & 5 \\ \text { Afraid of its toxicity } & 2 & 10 \\ \text { ITNs too hot to sleep under it } & 7 & 35 \\ \text { Housing structure affects ITN use } & 6 & 30\end{array}$

Children under 5 years age slept under ITN in previous night $(n=540)$

$\begin{array}{lll}\text { No } & 122 & 22.6 \\ \text { Yes } & 418 & 77.4\end{array}$

Pregnant women slept under ITNs previous night (72)

\begin{tabular}{lll} 
No & 18 & 25 \\
Yes & 54 & 75 \\
\hline
\end{tabular}

Table 4 Condition of ITNs in households who owned ITNs, in Chewaka district, South West Ethiopia, 2014

1.8 persons in malaria-endemic areas $<2000 \mathrm{~m}$ ) [15]. Hence, to attain sustainable control of the disease, households in the study area require extra nets to reduce the occupant per net gap.

The mode of malaria transmission identified by $59.6 \%$ of respondents in this study was mosquito bites. This result is higher than the findings from a study in Wonago Woreda, Southern Ethiopia, where $42.3 \%$ of respondents also listed mosquito bites [18]. But, less compared to a study done in Oromia and Amhara regional state, Ethiopia (67.9 \%) [19]. Respondents' perception of net use as a main preventive measure for malaria was also lower compared to a survey done in Southern Ethiopia [18]. The reason for this may be that the majority of respondents included in our study were from rural areas and had less access to health information. Nonetheless, about three quarters of our respondents had ever heard educational messages about mosquito nets, which is high 
Table 5 Final logistic regression model for household's ITNs use in Chewaka settlement, South West Ethiopia, 2014

\begin{tabular}{|c|c|c|c|c|}
\hline \multirow[t]{2}{*}{ Variables } & \multicolumn{2}{|c|}{ ITN Utilization Status } & \multirow[t]{2}{*}{ Crude OR (95 \% CI) } & \multirow[t]{2}{*}{ Adjust OR (95\%) Cl } \\
\hline & No & Yes & & \\
\hline \multicolumn{5}{|l|}{ Sex } \\
\hline Female & 61 & 196 & 1.00 & 1.00 \\
\hline Male & 22 & 138 & $1.95(1.14-3.33)$ & $1.60(0.87-2.94)$ \\
\hline \multicolumn{5}{|l|}{ Age (years) } \\
\hline $15-29$ & 31 & 175 & 1.00 & 1.00 \\
\hline $30-44$ & 40 & 136 & $0.60(0.36-1.01)$ & $0.59(0.32-1.07)$ \\
\hline$\geq 45$ & 12 & 23 & $0.33(0.15-0.75)$ & $0.54(0.20-1.42)$ \\
\hline \multicolumn{5}{|c|}{ Knowledge of malaria transmission by mosquito bites } \\
\hline No & 35 & 40 & 1.00 & 1.00 \\
\hline Yes & 48 & 294 & $5.36(3.10-9.26)$ & $3.44(1.80-6.59)^{*}$ \\
\hline \multicolumn{5}{|c|}{ Number of ITNs freely supplied for HHs } \\
\hline One/HH & 30 & 174 & 1.00 & 1.00 \\
\hline $\mathrm{Two} / \mathrm{HH}$ & 38 & 131 & $0.59(0.35-1.01)$ & $0.77(0.43-1.40)$ \\
\hline Three and above/HH & 15 & 29 & $0.33(0.16-0.69)$ & $0.80(0.33-1.92)$ \\
\hline \multicolumn{5}{|l|}{ Age of ITNs } \\
\hline$<1$ year & 49 & 139 & 1.00 & 1.00 \\
\hline $1-2$ years & 18 & 108 & $2.11(1.16-3.84)$ & $0.72(0.33-1.57)$ \\
\hline$>3$ years & 16 & 87 & $1.92(1.03-3.45)$ & $0.76(0.33-1.74)$ \\
\hline \multicolumn{5}{|l|}{ ITNs ever been washed } \\
\hline No & 42 & 70 & 1.00 & 1.00 \\
\hline Yes & 41 & 264 & $3.86(2.33-6.40)$ & $2.66(1.35-5.26)^{*}$ \\
\hline \multicolumn{5}{|c|}{ Is under five child/ children in the $\mathrm{HH}$} \\
\hline No & 18 & 41 & 1.00 & 1.00 \\
\hline Yes & 65 & 293 & $1.98(0.07-3.66)$ & $1.35(0.65-2.81)$ \\
\hline
\end{tabular}

* $P<0.05$ - Significantly associated

compared with national survey results (41.0\%) [20]. This may be because our study included only households who were freely supplied with ITNs, which would contribute to increased exposure to educational messages. The difference might also be due to the presence of extensive promotion of ITNs currently underway in the country.

Presence of holes/tears on nets was also associated with malaria infection. Around $79 \%$ of ITNs had holes/ tears $\geq 2 \mathrm{~cm}$, which is higher than a study done in Tanzania in which $45 \%$ of ITNs had holes/tears $\geq 2 \mathrm{~cm}$ [21]. It is also higher than a survey conducted in Malawi, where $12.8 \%$ of owners reported that nets had holes $>2 \mathrm{~cm}$ [22]. The reasons for this may include structural issues in the house that pose challenges to hang nets, such that the nets are too short to fully cover sleeping areas, long duration in use of ITNs, and frequent washing on rough surfaces.

Our finding revealed that there was strong association between using ITNs and knowledge of malaria transmission by bite of mosquito, which is in line with a study done Ghana [23, 24]. Households with ITNs ever been washed shows significant increase of utilization compared to households with unwashed ITNs. This might be due to households having been encouraged to use clean ITNs as opposed to dirty ITNs.

About $20 \%$ of ITNs were not used by households and $29 \%$ of ITNs were used for other purposes such as storage of sorghum, to make fences, protect bulls from insect bites. This implies that distribution of nets to communities without health education on the importance of ITNs in prevention of malaria, as well as how to use nets, may not bring about the desired result. In this study education levels of respondents, and ever heard education messages about ITN were not associated with the use of ITNs. Age and sex of respondents were not significantly associated with ITN utilization in this study. Some studies reported similar findings [25, 26], while the others showed significant associations between these factors and ITN use $[8,27]$. In addition, education levels and income did not significantly affect ITN use in our study which is in line with previous study done 
Ethiopia [8], Uganda [28] and Nigeria [29]. Given the cross-sectional nature of the results, interpretation of study results is limited. One of the major limitations of this study was that it relied on reported use of ITNs by households prior nights, without any means observation. Thus, the percentage of ITN use in this study might be overestimated due to self- reporting bias. In the future, research using a prospective cohort study design would be valuable.

\section{Conclusions}

This study in Southwest Ethiopia showed a high proportion of net ownership compared to a household survey from Ethiopia which included in the World Malaria Report. Despite somewhat high percentages ITN ownership, the study demonstrated there was still a gap between ownership and use of ITNs. On average, four individuals shared a single ITN and over one third of ITNs had at least one hole/tear. The two factors strongly associated with ITN use in surveyed households included knowledge of mosquito bites as a main mode of malaria transmission, and washing of ITNs at least once. To achieve sustained control of malaria, household coverage of nets alone is not sufficient. Public health interventions should also address problems related to utilization and care of ITNs. Intensive health education and community mobilization efforts should be employed to influence the specific factors identified as affecting ITN use.

\section{Competing interests}

The authors declare that they have no competing interests.

\section{Authors' contributions \\ TSB participated in the design of the survey, trained the research team and oversaw the fieldwork and critical revision of the manuscript. DO and BT trained the research team, oversaw the fieldwork and participated in editing the manuscript. All authors read and approved the final version of the manuscript.}

\begin{abstract}
Acknowledgments
Our sincere thanks go to the Mettu University for providing financial support to this study. We are grateful to the Ilu Aba Bor Zonal Health Office and Chewaka district Health Office for their cooperation and for giving us all the invaluable information we requested. The authors are grateful to Victoria Ryan for proof reading of the manuscript. Finally, we offer our gratitude to the study participants, as well as the field supervisors, data collectors and all others who made this study possible.
\end{abstract}

\section{Author details}

'Department of Public Health, Faculty of Public Health and Medical Sciences, Mettu University, Mettu, PO Box 318, llu Ababor, Ethiopia. ${ }^{2}$ Department of Midwifery, Faculty of Public Health and Medical Sciences, Mettu University, Mettu, PO Box 318, llu Ababor, Ethiopia.

Received: 23 February 2015 Accepted: 22 January 2016

Published online: 01 February 2016

\section{References}

1. Lozano R, Naghavi M, Foreman K, Lim S, Shibuya K, Aboyans V, et al. Global and regional mortality from 235 causes of death for 20 age groups in 1990 and 2010: a systematic analysis for the Global Burden of Disease. Study 2010. Lancet. 2012:380(9859):2095-128.

2. Nsungwa-Sabiiti J, Peterson S, Pariyo G, Ogwal-Okeng J, Petzold MG, Tomson G. Home-based management of fever and malaria treatment practices in Uganda. Trans R Soc Trop Med Hyg. 2007;101:1199-207.

3. Federal Democratic Republic of Ethiopia. National malaria guidelines. Third edition. Ministry of Health. Addis Ababa, 2012.

4. Alemu A, Abebe G, Tsegaye W, Golassa L. Climatic variables and malaria transmission dynamics in Jimma town, South West Ethiopia. Malar. J. 2011; 4(30). http://www.ncbi.nlm.nih.gov/pmc/articles

5. WHO. Insecticide-Treated Mosquito Nets: a WHO Position Statement. Geneva: World Health Organization; 2007.

6. Lengeler $\mathrm{C}$. Insecticide-treated bed nets and curtains for preventing malaria, Cochrane Database of Systematic Reviews 2004. 2009.

7. Eisele TP, Larsen D, Steketee RW. Protective efficacy of interventions for preventing malaria mortality in children in plasmodium falciparum endemic areas. Int J Epidemiol. 2010;39:188i101.

8. Loha E, Tefera K, Lindtjørn B. Freely distributed bed-net use among Chano Mille residents, south Ethiopia: a longitudinal study. Malaria Journal. 2013;12: 23. doi:10.1186/1475-2875-12-23.

9. Net Mark research 1999-2006. Available at: http://www.netmarkafrica.org/ research. (Accessed on: 15/08/ 2006).

10. Macintyre $K$, Keating J, Okbaldt YB. Rolling out insecticide treated nets in Eritrea: examining the determinants of possession and use in malarious zones during rainy seasons. Trop Med Int Health. 2006;11(6):824-33.

11. Koreromp EL, Miller J, Cibulskis RE, Kabir Cham M, Alnwick D, Dye C. Monitoring mosquito net coverage for malaria control in Africa: possession versus use by children under 5 years. Trop Med Int Health. 2003;8(8):693-703.

12. Tsuyuoko R, Midizi SM, Dziva P, Makunike B. The acceptability of insecticide treated mosquito nets among community members in Zimbabwe. Cent Afr J Med. 2002:48(7-8):87-91.

13. Astatkie A, Feleke A. Utilization of insecticide treated nets in Arbaminch Town and the malarious villages of Arbaminch Zuria District, Southern Ethiopia. Ethiop J Health Dev. 2009;23(3):206-15.

14. Roll Back Malaria, MEASURE Evaluation, World Health Organization, and UNICEF. Guidelines For Core Population Coverage Indicators For Roll Back Malaria: To Be Obtained From Household Surveys, MEASURE Evaluation, Calverton, Md, USA, World Health Organization; 2006

15. WHO. World Malaria Report. Geneva: World Health Organization; 2011.

16. RBM. The African Summit on Roll Back Malaria, Abuja, Nigeria. WHO/CDS/ RBM/200.17.RBM. Geneva: World Health Orginization; 2000, p. 556-6.

17. World Health Assembly. Fifty-Eight World Health Assembly: Malaria Control. In vol Resolution 55/284. Geneva: World Health Organization; 2005

18. Dagne G, Deressa W. Knowledge and utilization of insecticide treated mosquito nets among freely supplied households in Wonago Woreda, Southern Ethiopia. Geneva: Ethiop J Health Dev. 2008;22(1):34-41.

19. Baume AC, Reithinger $R$, Woldehanna S. Factors associated with use and non-use of mosquito nets owned in Oromia and Amhara Regional States, Ethiopia. Malaria Journal. 2009;8:264. doi:10.1186/1475-2875-8-264

20. Jima D, Tesfaye G, Deressa W, Woyessa A, Kebede D, Alamirew D. Baseline survey for the implementation of ITNs for malaria control in Ethiopia. Ethiop J Health Dev. 2005:19(1):16-23.

21. Erlanger $T$, Enayati A, Hemingway J, Mashinda $H$, Tami A, Lengeler $C$. Field issues related to effectiveness of insecticide treated nets in Tanzania. Med Vet Entomol. 2004;18(2):153-60.

22. Holtz TH, Marum LH, Mkandala C. ITN use, anemia, and malaria parasitemia in Blantyre district, Malawi. Trop Med Int Health. 2002;7(4):220-30.

23. Agyepong IA, Manderson L. Mosquito avoidance and bed net use in the Greater Accra Region, Ghana. J Biosoc Sci. 1999;31(1):79-92.

24. Binka FN, Adongo P. Acceptability and use of insecticide impregnated bednets in northern Ghana. Trop Med Int Health. 1997;2(5):499-507.

25. Berie $Y$, Alemu K, Belay A, Gizaw Z. Factors affecting utilization of Insecticide treated nets among people living with HIV/AIDs in Bahir Dar city, northwest Ethiopia. J Clin Med. 2013;2(6):147-52

26. Atieli E, Guofa Zhou G, Afrane Y, Lee M, Mwanzo I, Githeko K, et al. Insecticide-treated net (ITN) ownership, usage, and malaria transmission in the highlands of western Kenya. Parasit Vectors. 2011:4:113.

27. Aigbe W, Iwara O, Okongor E, Okino I. Ownership and utilization of Insecticide Treated nets in Cross River States, Nigeria. J Med Sci. 2012;12(7):198-206. 
28. Sangare LR, Weiss NS, Brentlinger PE, Richardson BA, Staedke SG, Kiwuwa $M S$, et al. Determinants of use of insecticide treated nets for the prevention of malaria in pregnancy: Jinja, Uganda. PLoS ONE. 2012;7, e39712.

29. Ye Y, Patton E, Kilian A, Dovey S, Eckert E. Can universal insecticide-treated net campaigns achieve equity in coverage and use? The case of northern Nigeria. Malar J. 2012;11:32

Submit your next manuscript to BioMed Central and we will help you at every step:

- We accept pre-submission inquiries

- Our selector tool helps you to find the most relevant journal

- We provide round the clock customer support

- Convenient online submission

- Thorough peer review

- Inclusion in PubMed and all major indexing services

- Maximum visibility for your research

Submit your manuscript at www.biomedcentral.com/submit 